Review

\title{
Dynamical Manipulation of Surface Plasmon Polaritons
}

\author{
Sen Wang *, Chunying Zhao and Xing Li * \\ Shandong Provincial Engineering and Technical Center of Light Manipulations \& Shandong Provincial Key \\ Laboratory of Optics and Photonic Device, College of Physics and Electronics, Shandong Normal University, \\ Jinan 250014, China \\ * Correspondence: wangsensdnu@sdnu.edu.cn (S.W.); lixing0607@126.com (X.L.)
}

Received: 12 July 2019; Accepted: 8 August 2019; Published: 11 August 2019

\begin{abstract}
As the fundamental and promising branch of nanophotonics, surface plasmon polaritons (SPP) with the ability of manipulating the electromagnetic field on the subwavelength scale are of interest to a wide spectrum of scientists. Composed of metallic or dielectric structures whose shape and position are carefully engineered on the metal surface, traditional SPP devices are generally static and lack tunability. Dynamical manipulation of SPP is meaningful in both fundamental research and practical applications. In this article, the achievements in dynamical SPP excitation, SPP focusing, SPP vortex, and SPP nondiffracting beams are presented. The mechanisms of dynamical SPP devices are revealed and compared, and future perspectives are discussed.
\end{abstract}

Keywords: surface plasmon polaritons; dynamical modulation; excitation; focusing; vortex; nondiffracting beam

\section{Introduction}

Arising from the collective oscillations between the incident phonons and electrons at the dielectric/metal interface, surface plasmon polaritons (SPP) exhibit intriguing properties of large in-plane momentum, subwavelength field confinement, and strong field enhancement [1-3], which makes it promising in applications ranging from sensors [4], solar cells [5], super-resolution imaging [6-8], optoelectronic circuits [9-11], and more. Modulating the propagation and distribution of SPP is indispensible in these applications. Most optical devices in the 3D free space can find their $2 \mathrm{D}$ counterparts in the SPP platform. SPP focusing [12-17], SPP refection [18-20], SPP vortex [21-24], SPP Airy beam [25-27], SPP Bessel beam [28-30], SPP logic gates [31,32], and unidirectional SPP excitation [33-35] have been accomplished by elaborately designing the shape and position of dielectric or metallic structures. Nevertheless, the functionalities of SPP devices are generally single and static.

Dynamic light modulation is always desired in different branches of optics. In the free space, the amplitude and phase of light can be readily modulated with spatial light modulator (SLM). For SPPs, however, no such modulator exists. Fortunately, the amplitude and phase of SPPs are closely related to the excitation light. The amplitude of SPPs is proportional to the intensity of the incident light and the phase is the same as that of the incident light. Based on this feature, the distribution of SPPs can be dynamically manipulated by modulating the excitation light [36-45]. Another distinctive feature is that the amplitude and phase of the excited SPPs depend heavily on the polarization states of incident light. Particularly, for circularly polarized light, the SPPs generated by subwavelength slits are imprinted with a spin-dependent Pancharatnam-Berry (PB) phase which can be tuned by changing the orientation angle of the slits [46,47]. Polarization-controlled dynamical SPP excitation [48-56], SPP focusing [46,57-66], SPP vortex [21,44,67-73], SPP nondiffracting beams [74-76], and SPP holography [77,78] have been 
realized. Besides, the other degrees of freedom of light-including the wavelength, topological charge, and the spatial frequency - can be taken advantage of to actively control the SPP field [79-86] as well.

In this paper, we review the achievements in a branch of plasmonic-dynamical SPP modulation. In the main part, different approaches adopted to actively manipulate SPP excitation, SPP focusing, SPP vortex, and SPP nondiffracting beams are explained. The underlying physics of dynamical SPP devices are summarized and comparisons of the different approaches are presented in Section 6. In passing, the basic properties of SPPs and the detection of SPP fields which can be found in [1-3] are not involved in this review. The non-equilibrium properties of localized surface plasmon generated around single metal nanoparticle, such as ultra-fast nonlinear dynamics $[87,88]$ are not included either.

\section{Directional Excitation of Surface Plasmon Polaritons (SPP)}

\subsection{Polarization Controlled Excitation of SPP}

SPP source is the fundamental component of 2D plasmonic system. Slits and gratings are the commonly used structure to satisfy the wave vector matching condition and generate SPP $[1,2]$. Conventionally, the excitation of SPP is bidirectional and symmetrical. Dynamic control of the propagation direction of SPP is desired for real applications. Figure 1a shows polarization controlled directional SPP coupler which consists of two parallel columns of orthogonal rectangle slits with separations [48]. SPP waves propagating to the left and right are the interference of the SPP launched by each column, which can be expressed as

$$
\begin{aligned}
& I_{r} \propto\left[\left(I_{1}+I_{2}\right)+2 \sqrt{I_{1} I_{2}} \cos \left(k_{s p} s+\delta\right)\right], \\
& I_{l} \propto\left[\left(I_{1}+I_{2}\right)+2 \sqrt{I_{1} I_{2}} \cos \left(k_{s p} s-\delta\right)\right],
\end{aligned}
$$

where $I_{1}$ and $I_{2}$ are the intensities of SPP generated by the two columns of slits, $k_{s p}$ is the wave vector of SPP. $\delta$ is relative phase between the two orthogonal components of incident light, which determines the polarization state. When the separation $s$ is adjusted to $s=\lambda_{s p} / 2$, Equation (1) can be simplified to

$$
\begin{aligned}
& I_{r} \propto\left[\left(I_{1}+I_{2}\right)-2 \sqrt{I_{1} I_{2}} \sin \delta\right] \\
& I_{l} \propto\left[\left(I_{1}+I_{2}\right)+2 \sqrt{I_{1} I_{2}} \sin \delta\right]
\end{aligned} .
$$

From Equation (2), it can be concluded that the overall intensity of excited SPP $I=I_{1}+I_{2}$ is independent of relative phase, but the proportion of SPP propagating to the left or right can be tuned by changing polarization state. Particularly, with the illumination of circularly polarized light, $I_{1}$ and $I_{2}$ are equal and the relative phase is $\delta=\sigma_{ \pm} \frac{\pi}{2}$, where $\sigma_{ \pm}= \pm 1$ represents left circularly polarized (LCP) and right circularly polarized (RCP) light, respectively. Thus, SPP excited by LCP light will propagate to the left $\left(I_{r}=0\right)$ and SPP excited by RCP light will propagate to the right $\left(I_{l}=0\right)$. For linearly polarized light which can be regarded as the combination of LCP and RCP light, the excited SPPs propagate equally to the left and right side. The counterclockwise, clockwise, and linear arrows in Figure 1a indicate the polarization stats of the incident light. The experimental results further demonstrate that controlled directional SPP excitation is possible by changing the handedness of the incident light [48]. Based on anomalous diffraction, a similar functionality is realized with a metasurface consisting of spatial-variant subwavelength slits [49], as shown in Figure 1b. Light normally incident on the metasurface will experience both ordinary and anomalous diffraction. The orientation angle $\alpha$ of the slits has no effect on the ordinary diffracted light and the metasurface acts as a grating, while the anomalous diffracted light is imprinted with a spin-dependent phase $2 \alpha$. As a result, the anomalous diffraction beam patterns are not symmetrical about the surface normal, which makes it possible to realize directional SPP excitation. To excite SPP, the in-plane wave vector of diffracted light should satisfy.

$$
k_{s p}=\frac{2 m \pi}{s}+\sigma_{ \pm} \frac{2 \Delta \alpha}{s} .
$$


For LCP incident light with a frequency of $\omega_{2}$, the SPPs generated by the $m=1$ anomalous diffraction is shifted to a higher frequency $\omega_{1}$ and will propagate to the left, while the SPPs generated by the $m=-1$ anomalous diffraction are shifted to a lower frequency $\omega_{3}$ and will propagate to the right. When the handedness of the incident light is switched, the propagation direction of SPPs will be reversed correspondingly. The phase gradient $\Delta \alpha$ inducing extra in-plane momentum accounts for the polarization-controlled SPP excitation.

(a)

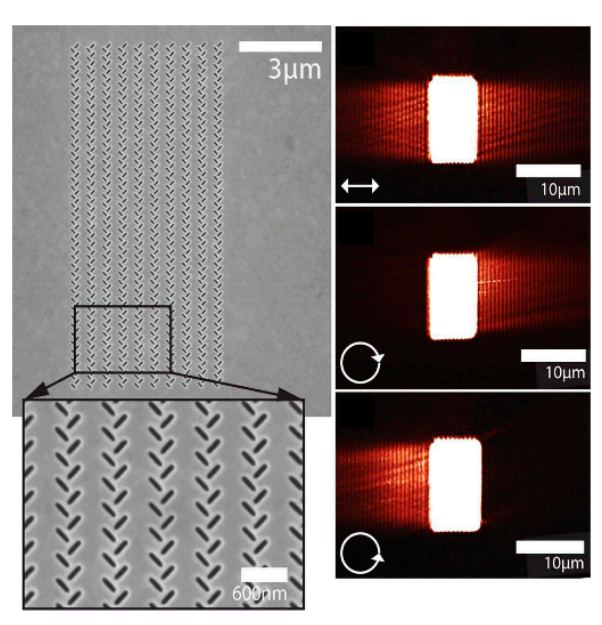

(b)

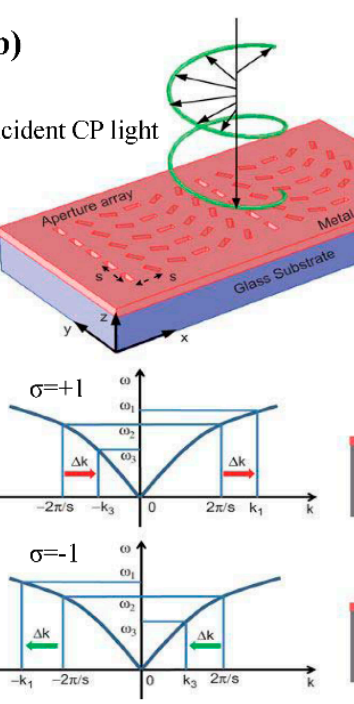

(c)
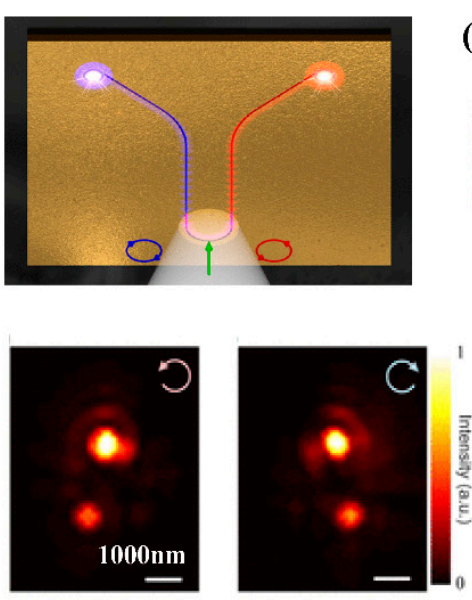

(d)
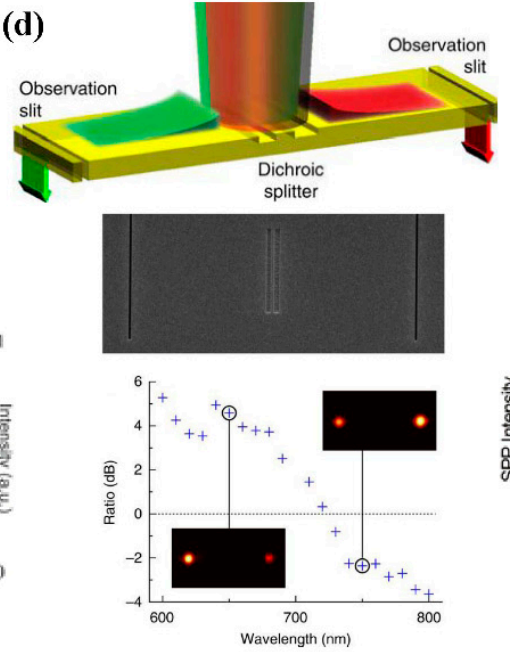

Incident $\sigma=1 \psi$

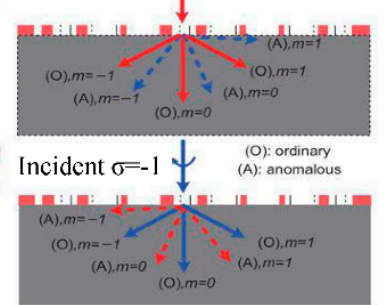

Incident $\mathrm{CP}$

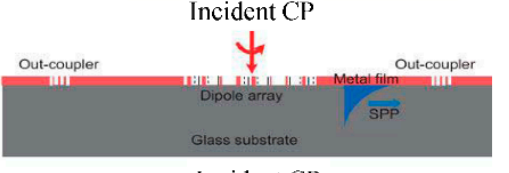

Incident CP

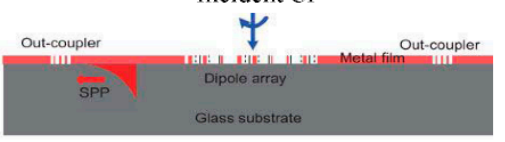

(e)
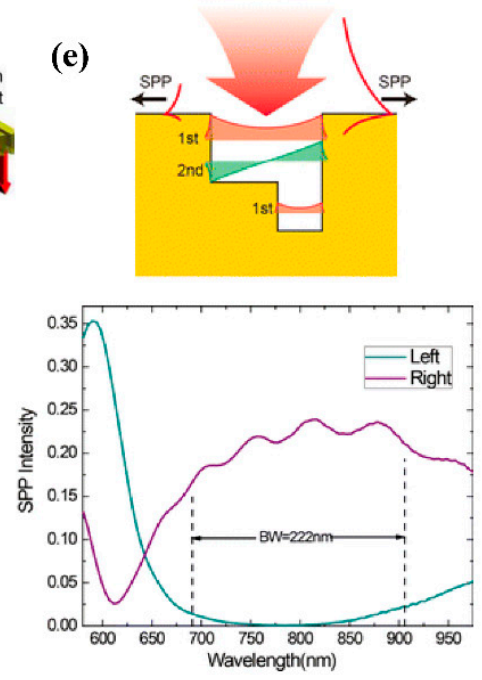

Figure 1. (a) Polarization controlled directional excitation of surface plasmon polaritons (SPPs) [48]; (b) Directional SPP excitation with interfacial phase discontinuity [49]; (c) Directing SPP flows based on spin and orbit angular momentum interaction [50]; (d) Wavelength dependent directional SPP excitation [79]; (e) Broadband directional SPP excitation with a single compact antenna [80]. Reproduced with permissions from: [48], AAAS, 2013, [49], Springer Nature, 2013, [50], ACS, 2018, [79], Springer Nature, 2011, and [80], ACS, 2015.

Figure 1c schematically shows a curved plasmonic waveguide which can realize directional SPP propagation at a deep subwavelength scale [50]. The circular slit provides an extrinsic orbital angular momentum (OAM) $[89,90]$ to match the spin angular momentum (SAM) of incident phonon, which enables the spin-orbit interaction (SOI). Two counter propagating modes can be sustained in such a 
curved single-mode waveguide and, considering the longitudinal angular momentum conservation, the corresponding probabilities of the two modes can be expressed as

$$
\begin{aligned}
& \left|a_{r}\right|^{2}=\frac{1+\sigma_{ \pm} / \beta R}{2} \\
& \left|a_{l}\right|^{2}=\frac{1-\sigma_{ \pm} / \beta R}{2} .
\end{aligned} .
$$

$\beta$ is the wave vector of the waveguide modes and $R$ is radius of the circular waveguide. When $\beta$ satisfies the geometric dynamical condition $(\beta=\sigma / R)$, the propagation direction of the surface plasmon is determined by the handedness of incident light. In the experiment, the operation wavelength is $1.55 \mu \mathrm{m}$ and the corresponding radius $R$ is $500 \mathrm{~nm}$. The results show that the directionality (the ratio of emitted intensity) of the subwavelength plasmonic network for both LCP and RCP light can reach 95\%.

\subsection{Wavelength Dependent Directional SPP Excitation}

Resembling the wavelength-division multiplexing in the 3D space, the wavelength demultiplexing of SPP is a feasible ingredient for on-chip optical communication. In Figure 1d, adopting two grooves with different widths, a submicron plasmonic dichroic splitter is demonstrated [79]. The SPP transmission and reflection features of the groove can be tuned by changing the depth and width. Based on numerical simulation, the two grooves are elaborately designed so that one groove mainly transmits the SPP and the other reflects SPP at a specific wavelength. For another wavelength, the functionalities of the two grooves are reversed. In the experiment, two grooves with widths of $260 \mathrm{~nm}$ and $360 \mathrm{~nm}$ and depths of $100 \mathrm{~nm}$ are fabricated. The ratio between the SPP propagating to the left and right is 3:1 at $640 \mathrm{~nm}$ and the ratio is reversed to 1:2 for $790 \mathrm{~nm}$ incident light. Based on mode conversion and mode interference, directional SPP excitation can also be achieved with a single compact antenna containing one groove and another auxiliary resonant groove [80], shown in Figure 1e. With normally incident light, the symmetric first waveguide modes (red) in both the main groove and lower nanogroove are excited. The first mode in the lower nanogroove propagates downward and is reflected by the bottom, which can activate the antisymmetric second mode (green) in the upper main groove. In this way, the auxiliary nanogroove enables the energy conversion as well as the interference between the two modes in the main groove. The amplitudes of the modes can be tuned by the height of lower nanogroove and the phase difference between the two modes is determined by the height of main groove. SPP generation by the compact antenna can be attributed to three factors: the normally incident beam, the first mode and the second mode in the main groove. The SPP propagating to the left and right are in phase for the first two factors, but are antiphase for the third factor. Thus, directional SPP coupler can be obtained by adjusting the SPP generated by three factors. In the experiment, the maximum intensity ratio between the rightward and leftward SPP is over $10^{3}$ around the central wavelength of $800 \mathrm{~nm}$. The directional SPP coupler works well with rather wide spectral range of $222 \mathrm{~nm}$. That is because the grooves are shallow and the corresponding phase varies slowly for the different modes. It should be noted the third mode in the main groove can be activated at the short wavelength region around $609 \mathrm{~nm}$, which can turn the propagation direction of SPP from right to the left.

\subsection{Directional SPP Excitation Determined by the Topological Charge of Vortex Beam}

When the incident light is vortex beam, the sign of the topological charge can also determine the propagation direction of SPP. As shown in Figure 2a, two gratings with different periods $\Lambda$ (denoted by the green and purple triangles) are utilized to compose the directional SPP coupler [82]. According to the wavevector matching condition, the reciprocal vector of grating $\vec{G}_{1,0}=2 \pi / \Lambda$, wavevector of the azimuth component of the incident beam $\vec{k}_{O A M}$ and $\vec{k}_{s p}$ should satisfy

$$
\vec{k}_{s p}=\vec{k}_{O A M}+\vec{G}_{1,0} .
$$


When the relation between the grating periods and the topological charge $l$ follow the curves in Figure 2a, the above wave vectors are collinear. From the analyses of the wave vectors, it can be seen that the wave vectors of SPP synthesized by the upper grating and the lower grating are the same and only the SPP propagating to the right satisfies Equation (5) for the $l=1$ incident vortex beam. If the topological charge is switched to $l=-1$, the direction of $\vec{G}_{1,0}$ will be reversed and SPP will propagates leftward. The experimental results in Figure 2a verify the feasibility of the directional SPP coupler.

(a)
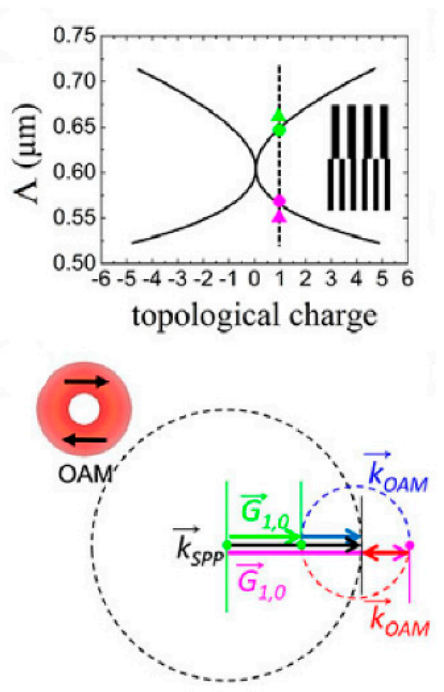

(b)

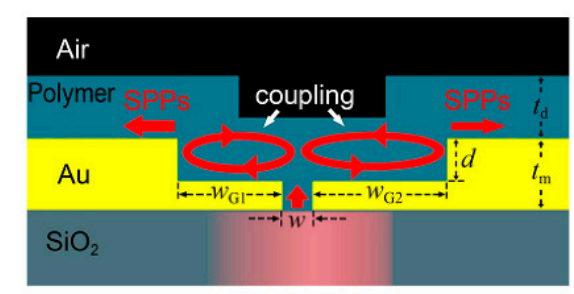

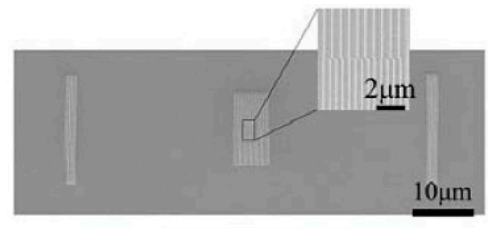
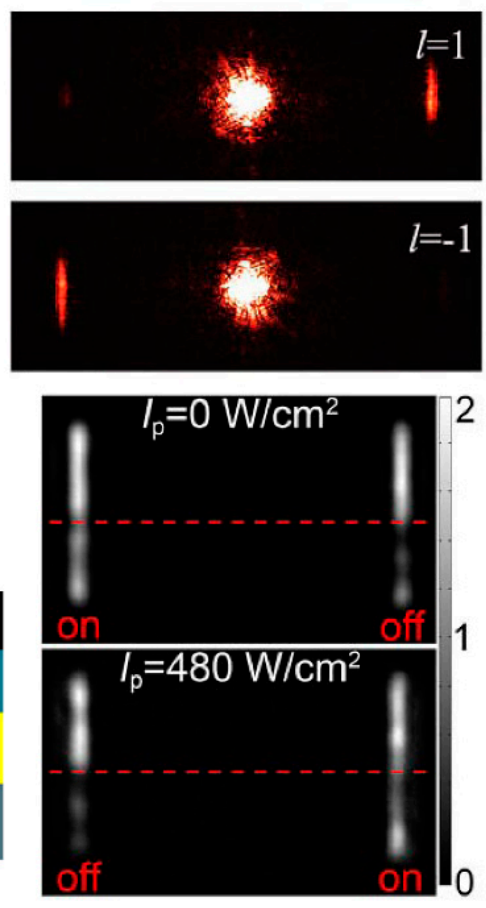

Figure 2. (a) Controlling the propagation direction of SPP by changing the topological charge of the incident vortex beam [82]; (b) Coating the slits with nonlinear polymer film, an all-optical directional SPP coupler can be achieved [91]. Reproduced with permissions from: [82], Wiley-VCH, 2018, [91], Springer Nature, 2013.

\subsection{Directional SPP Propagation Based on Nonlinear Optical Materials}

Nonlinear optical materials whose optical property strongly dependents on the pump light play vital roles in manipulating photons. Combing SPP and nonlinear optical materials can enrich the functionalities of SPP devices. Figure $2 b$ schematically shows the structure of the all-optical plasmonic switch, an asymmetric T-shape slit covered with nonlinear polymer film [91]. SPP generated by the slit propagate along the bottom of the grooves which can be regarded as Fabry-Pérot (FP) resonators. Without the pump beam, by carefully designing the length of FP resonators $\left(w_{G 1}=280 \mathrm{~nm}\right.$, $w_{G 2}=600 \mathrm{~nm}$ ), the SPP excited by $790 \mathrm{~nm}$ incident light can interfere constructively in one direction and interfere destructively in the other direction. When the slits are illuminated by the $532 \mathrm{~nm}$ pump beam, the refractive index of the polymer film is changed and the effective length of the FP resonator is changed as well. Thus, the propagation direction of SPP changes from left to right, which can be seen from the SPP distribution in Figure $2 b$. 


\section{Dynamical Focusing of SPP}

The initial studies about SPP focusing are done with circular or semicircular slits [13,14]. In 2008, Erez Hasman et al. found an interesting phenomenon that the SPP focused by semicircular slits would experience a spin-dependent transverse shift $[57,58]$, as shown in Figure 3a. The subwavelength scale displacement can be estimated with $\lambda_{s p} / 2 \pi$. In analogy with the spin Hall effect in the free space, this effect is attributed to a geometric spiral phase which unifies the spin redirection Berry phase and the PB phase. In 2015, we experimentally observed the spiral phase in the terahertz region and proposed an intuitive model based on Fourier transformation to illustrate this effect [60]. It should be noted that the transverse shift is smaller than the wavelength of SPP because of the limited spiral phase ranging from 0 to $\pi$. The circular or semicircular slits can be regarded as the constitution of subwavelength slits which are extensively utilized to design the optical metasurface. For a rectangle slit illuminated by circularly polarized light, the excited SPP field can be expressed as

$$
E_{s p}(r)=\sin \alpha \exp \left(i \sigma_{\mp} \alpha\right) \frac{\exp \left(i k_{s p} r+i \sigma_{ \pm} \pi / 2\right)}{\sqrt{r}}
$$

where $\alpha$ represent the orientation angle of the slit and $r$ is the distance from the slit [62]. Thus, the phase and amplitude of SPP can be modulated by changing the orientation angle of the slits. Moreover, the phase modulation depends on incident polarization. As shown in Figure 3b, two columns of slits with a distance $d$ are arranged according to the Fresnel zone plate to focus SPP [46] and the orientation angles are $\alpha_{0}$ and $-\alpha_{0}$, respectively. The phase of SPP propagating to the left and right can be written as

$$
\varphi_{s p}^{l}=2 \sigma_{ \pm} \alpha_{0}-k_{s p} d, \varphi_{s p}^{r}=2 \sigma_{ \pm} \alpha_{0}+k_{s p} d .
$$

When $\alpha_{0}=\pi / 4$ and $d=\lambda_{s p} / 4$, SPP will be solely focused on the left for LCP incident light $\left(\varphi_{s p}^{l}=0, \varphi_{s p}^{r}=\pi\right)$ and will be focused on the right for RCP light $\left(\varphi_{s p}^{l}=\pi, \varphi_{s p}^{r}=0\right)$. In the experiment, the focal length is $24 \mu \mathrm{m}$ and incident wavelength is $980 \mathrm{~nm}$, which indicates that the separation between the SPP focuses generated by LCP and RCP light is much larger than the wavelength.

Besides, considering that the spiral phase is the origin of spin-dependent SPP focusing for the semicircular lens in Figure 3a, introducing additional spiral phase should be able to amplify the transverse displacement. In Figure 3c, orthogonal slit pairs with spatial variant orientation angles are positioned along the semicircular circle [62]. The SPP field generated by an orthogonal slit pair is given by $E_{s p}(r)=-\exp \left(i \sigma_{\mp} 2 \alpha\right) \frac{\exp \left(i k_{s p} r\right)}{\sqrt{r}}$. The overall spiral phase contains two parts: the intrinsic spiral phase dependent on the handedness of incident light and the additional spiral phase caused by the rotation of slit pair, which can be written as $\varphi_{s p}=\sigma_{\mp}(\theta+2 \alpha)$. The separation between the two focuses generated by LCP and RCP light reaches $1500 \mathrm{~nm}$ for $632.8 \mathrm{~nm}$ incident light, which could reduce the crosstalk in on-chip communications.

For the semicircular SPP lens, SPPs excited by different wavelength incident light are always focused in the center, because the optical paths of SPPs are always equal to radius of the semicircle. A multiple-wavelength SPP lens is desirable to enhance the capacity of on-chip SPP systems. As shown in Figure $3 \mathrm{~d}$, the positions of the nanoslits are optimized with an iterative algorithm to design a compact triplexer [81]. The different positions of the slits correspond to the phase modulation of SPP. In the experiment, $5 \times 6$ nanoslits are adopted and the positions of the SPP focuses generated by $820 \mathrm{~nm}$, $850 \mathrm{~nm}$, and $880 \mathrm{~nm}$ incident light are $(0,-3 \mu \mathrm{m}),(0,0)$, and $(0,3 \mu \mathrm{m})$, respectively. Simultaneously controlling the polarization and the wavelength of incident light can enable us to manipulate the SPP focus more flexibly. The wavelength determines the positions of the SPP focuses and the polarization plays the on/off role [64], for the SPP lens in Figure 3e. The SPP lens consists of horizontal nanoslits and vertical nanoslits which can be correspondingly excited by vertically polarized and horizontally polarized light, because the polarization component parallel to the nanoslits cannot effectively give rise to SPP. In the design, four imaginary SPP source points are placed in different positions and the 
different color circles represent the equiphase lines. Vertical nanoslits are placed at the intersections of equiphase lines for $632 \mathrm{~nm}$ and $670 \mathrm{~nm}$ incident light, denoted by the black dots, while horizontal nanoslits are placed at the intersections of equiphase lines for $710 \mathrm{~nm}$ and $750 \mathrm{~nm}$ incident light. Thus, for incident light with a particular wavelength and polarization, the SPP generated by the horizontal or vertical nanoslits are in phase and will interfere constructively at the corresponding positions of the imaginary source point.

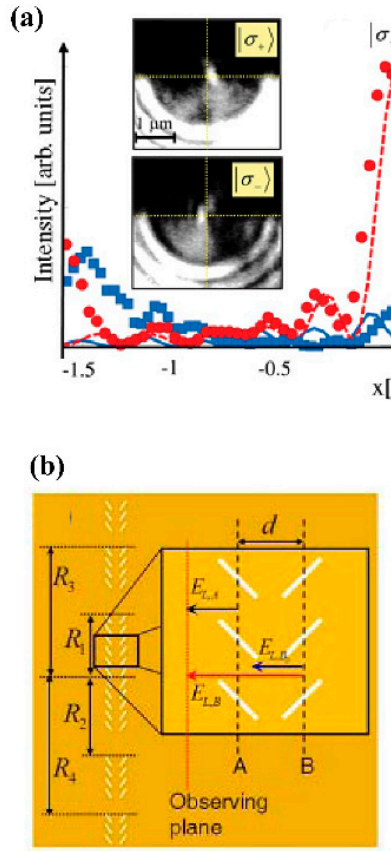

(c)

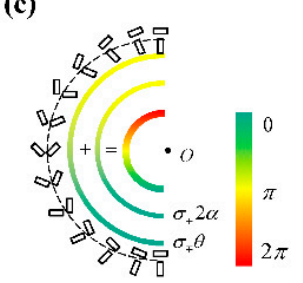

$\left|\sigma_{-}\right\rangle\left|\sigma_{+}\right\rangle$

\section{-}
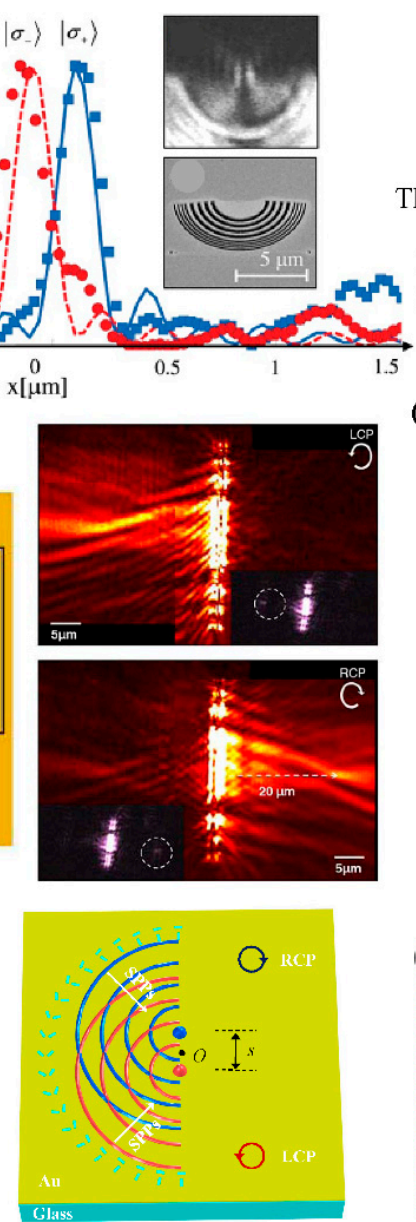

(e)
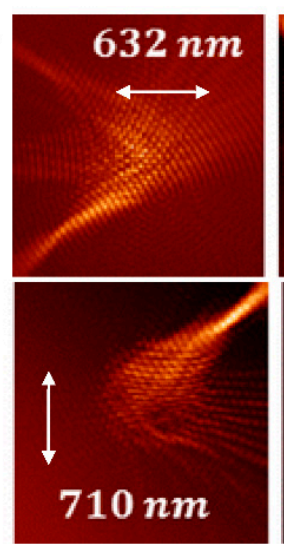

(d)
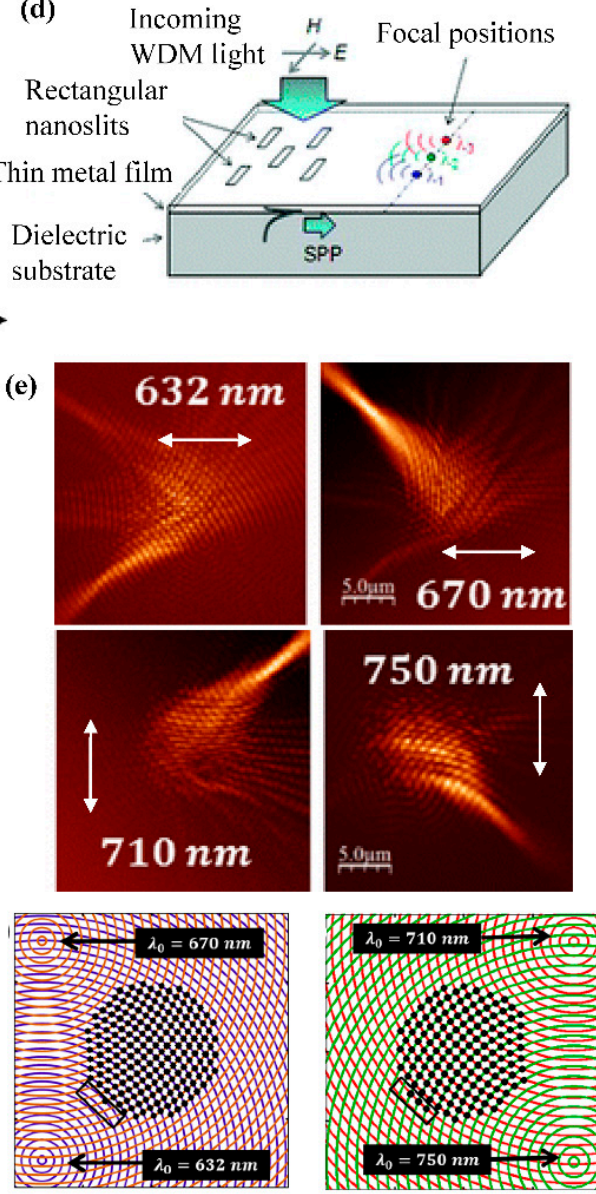

Figure 3. (a) Polarization-controlled focusing of SPP with semicircular slits [58]; (b) Spin-selected SPP focusing with vertically arranged nanoslits [46]; (c) Enlarging the spin-dependent transverse shift of SPP focus by introducing additional spiral phase [62]; (d) Multiple-wavelength focusing of SPP with a nonperiodic nanoslit coupler [81]; (e)Switchable SPP focusing by changing either the wavelength or polarization of incident light [64]. Reproduced with permissions from: [58], APS, 2008, [46], OSA, 2015, [62], OSA, 2019, [81], ACS, 2011, [64], ACS, 2015.

As shown in Figure 4a, a semicircular SPP lens is illuminated by the vortex incident beam with helical wavefront, and the position of SPP focus will be determined by topological charge $l$ of the vortex beam [83]. The spiral phase carried by the vortex beam can be transferred to the excited SPP. Thus, the phase of SPP can be expressed as $\varphi_{s p}=\left(\sigma_{ \pm}+l\right) \theta, 0 \leq \theta \leq \pi$. Just like the focusing of SPP excited by circularly polarized light in Figure 3a, the SPP focuses generated by vortex beams also experience transverse shifts. For a vortex beam with a larger topological charge, the spiral phase is larger and the displacement will also be larger. Figure 4 a shows the experimentally measured SPP distributions for vortex incident beams with different topological charges. The markers $l=1,2,3,4$, 5 represent the different topological charges and a white line is drawn along the $y$-axis to show the displacements of the SPP focuses. It can be clearly seen that the transverse shift of SPP focus grows 
with the increase of the topological charge. The interval between the SPP focuses generated by two neighboring topological charges is about $120 \mathrm{~nm}$ for $633 \mathrm{~nm}$ incident light. This feature can be utilized to discriminate the topological charge of incident light by measuring the displacement of SPP focus. From the above analyses, it can be concluded that the phase of excitation light can greatly influence the distribution of SPP. Thus, controlling the incident light with a spatial light modulator (SLM) can actively modulate the focusing of SPP [36], as shown in Figure 4b. The required phase to focus SPP at a pre-chosen position is obtained with an optimization loop and is then projected onto the metallic nanohole array through lenses $L_{1}$ and $L_{\mathrm{obj}}$. The distribution of SPP is imaged on to the camera through lenses $L_{\mathrm{obj}}$ and $L_{2}$. Experimental results show that SPP can be focused at target positions (center or corner) without mechanical motion. Amplitude modulation of excitation light with a SLM can also implement dynamical SPP focusing [37]. Fresnel-zone type binary amplitude images are projected onto a metallic grating. The focal length of SPP is given by $f=\left(4 r_{m}^{2}-m^{2} \lambda_{s p}^{2}\right) / 4 m \lambda_{s p}$, where $m$ is the maximum order number and $r_{m}$ is the radius of $m$ th zone. The odd-order zones are illuminated by incident light and can give rise to SPP, while the even-orders are not illuminated. Thus, the focal length of SPP can be tuned by changing $m$ and $r_{m}$. Changing the effective index of SPP propagating along the metal surface can also modulate the focusing property of SPP lens [92].

(a)
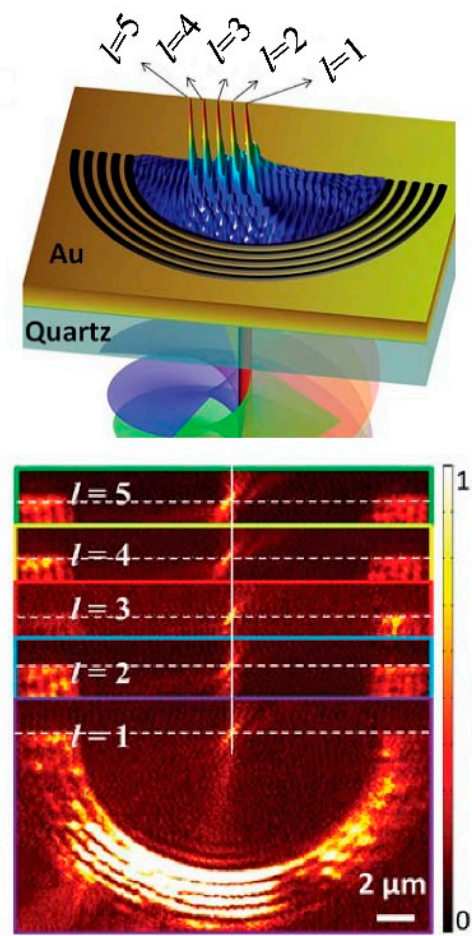

(c)

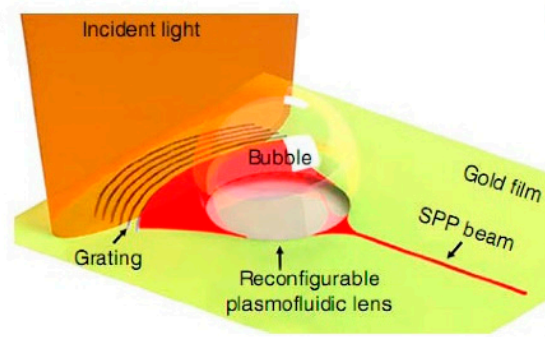

(b)
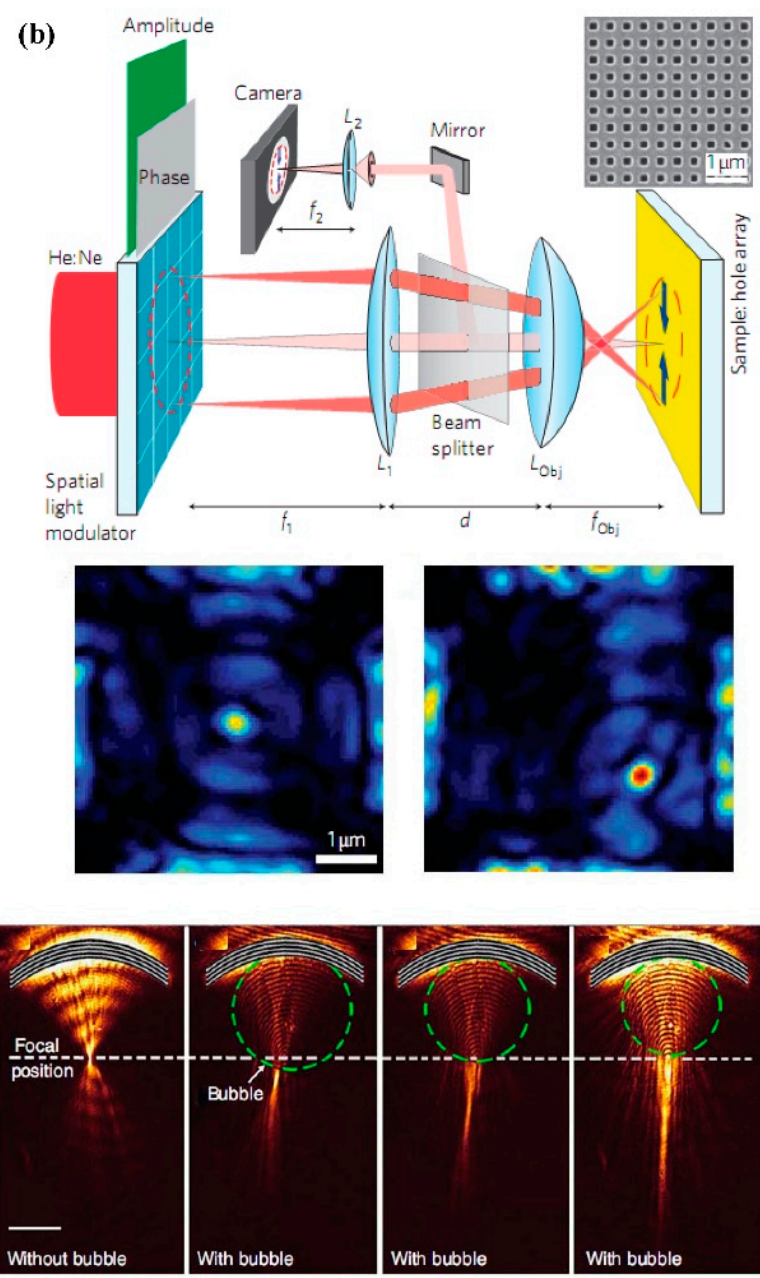

Figure 4. (a) Controlled focusing of SPP with vortex incident beam [83]; (b) Adjusting the SPP focus by modulating the phase and amplitude of the incident light with a spatial light modulator (SLM) [36]; (c) A dynamically tunable and reconfigurable SPP lens immersed in the water [92]. Reproduced with permission from: [83], RSC, 2016, [36], Springer Nature, 2011, [92], Springer Nature, 2013. 
In Figure 4c, an arc-shaped grating SPP lens is immersed in the water. Besides the $808 \mathrm{~nm}$ incident light used to excite SPP, another blue-violet diode laser with a wavelength of $405 \mathrm{~nm}$ is utilized to heat up the gold film and generate vapor bubbles on the surface because of the laser-induced thermal effect. The size and the location of the bubbles can be modulated by moving the laser spot and adjusting laser power. The generation of the bubbles causes the local changes in the effective index for SPP during propagation. The effective index of SPP inside the surface bubble (1.02) is smaller than that outside the bubble (1.379). Thus, the truncated sphere shape bubble functions like the conventional concave lens in the free space. Without the bubble, the SPP are focused in the center. When a bubble is generated and its size is decreased gradually, the SPP focus moves from the boundary of surface bubble to the outside and the profile of the focus evolves from a spot to a collimated beam, as can be seen from the experimental results in Figure 4c.

\section{SPP Vortex Generation}

With the helical wavefront, optical vortexes in free space are usually generated with spiral phase plates or SLMs $[89,90]$. Similarly, imposing spiral phases on SPP can also synthesis SPP vortexes. SPP generated by Archimedes spiral slits which are defined by $r_{m}(\theta)=r_{0}+\frac{\lambda_{s p} \bmod (m \theta, 2 \pi)}{2 \pi}$ are imprinted with a spiral phase along the slits because of the different optical path during propagation. The radius $r$ of slit changes with azimuthal angle $\theta$ and $m$ determines the partial slit segments and the topological charge of the generated SPP vortex. With the illumination of LCP and RCP light, Figure 5a schematically gives the phase of SPP generated by $m=2$ spiral slit [21,70]. It should be pointed out that there exists another spin-determined spiral phase besides the spiral phase induced by a different optical path. Therefore, the topological charge of the SPP vortex can be expressed by $j=m+\sigma_{ \pm}$. For an $m=4$ spiral slit, the topological charge of the SPP vortexes generated by LCP and RCP light are $j=5$ and $j=3$, respectively. The simulated SPP distributions in Figure 5a show that the diameter of the SPP vortex for the LCP light is larger than the one for RCP light, which is consistence with the theoretical analyses. When the spiral slits are illuminated by optical vortex beams as shown in Figure $5 b$, the spiral phase of incident vortex beam can be transferred to the SPP vortex. Hence, the total topological charge of the SPP vortex is given by $j=l+m+\sigma_{ \pm}[44,68]$. Numerical results in Figure $5 \mathrm{~b}$ give the profiles of SPP vortexes generated in different cases, which verifies that SPP vortex can be dynamically modulated by changing the polarization and the topological charge of the incident light. However, the maximum topological charge modulation of SPP vortex obtained by switching the handedness of the incident light is limited to $\Delta j= \pm 1$, because the spiral phases induced by the spiral structure and the incident vortex beam are polarization independent. As shown in Figure $5 c$, replacing the spiral slits with subwavelength nanoslits can overcome this restriction [73], considering that spin-dependent PB phase can be introduced by changing the orientation angles of the slits. The topological charge of the generated SPP vortex is $j=\sigma_{ \pm}(2 n-1)$, where $n$ is the ratio between the orientation angle $\alpha$ and the azimuthal angle $\theta$. Figure $5 \mathrm{c}$ schematically shows the structure of the $n=1.5 \mathrm{SPP}$ vortex generator, and the simulated phase distributions show that the topological charges are $j=2$ and $j=-2$ for LCP and RCP incident light, which agrees with the theoretical value. 
(a)
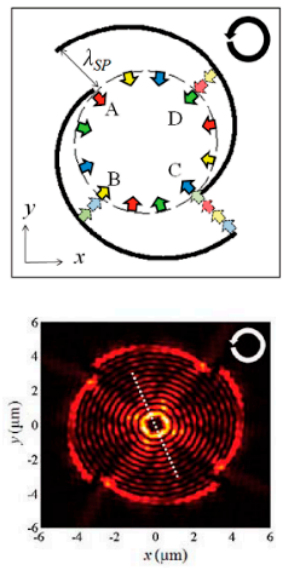
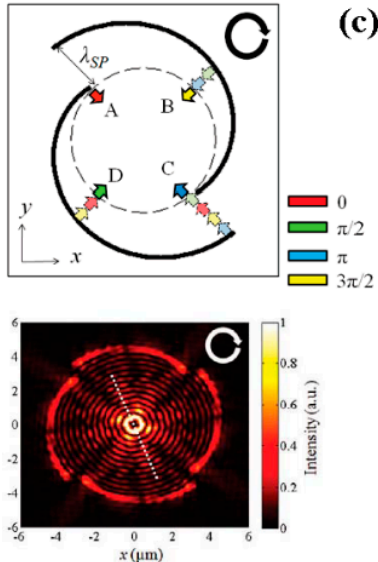

(c)
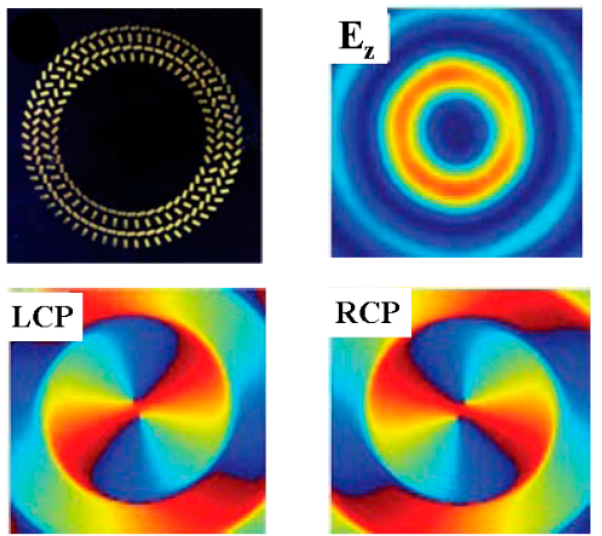

(b)

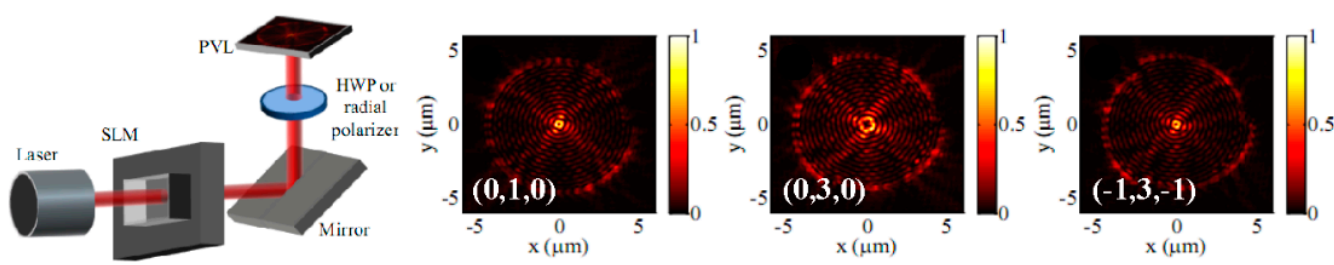

Figure 5. For Archimedes spiral slits, the topological charge of SPP vortex can be modulated by the polarization (a) or topological charge (b) of the incident light [21,44]. (c) For SPP vortex generator consisting of nanoslits, dynamically reversing the topological charge of the high-order SPP vortex can be realized [73]. Reproduced with permissions from: [21], ACS, 2010, [44], OSA, 2012, [73], Wiley-VCH, 2018.

\section{Nondiffracting SPP Beams}

Besides the attenuation caused by absorption of metal, the propagation of SPP also suffer from the scattering because of the rough surface and inherent diffraction. Nondiffracting SPP beams with the diffraction-free and self-healing properties can effectively overcome these effects and lengthen the propagating distance of SPP. Inspired by the generation of nondiffracting beam in the 3D free space, 2D plasmonic Airy beams and Bessel beams have been accomplished and extensively studied $[25,26,28-30]$. Actively controlling the trajectory, position, propagation direction, and profile of the nondiffracting SPP beams is realized as well. Figure 6a schematically exhibits the experiment setup used to dynamically manipulate the trajectory of SPP Airy beam [42]. 1D Airy beam which is generated through the Fourier transform of a Gaussian beam with a cubic phase is impinged on to a metallic grating and then the excited SPPs take on the profile of incident Airy beams. Mechanical displacements of objective lens $\mathrm{O}_{1}$ along the transversal or longitudinal direction can change the incident angle of the Airy beam, and an oblique phase is imprinted on the SPP Airy beam. Thus, the propagation direction of the SPP Airy beam can be adjusted by changing the incident angle. Likewise, controlling the displacements of the input Gaussian beam and the cubic phase can modulate the trajectory of the SPP Airy beam. With subwavelength nanoslits, the position of SPP Airy beam can be controlled by the incident polarization [74], as shown in Figure 6b. To generate SPP Airy beam, the length of the slits are designed according to the Airy function and a binary phase $(0$ and $\pi)$ modulation is adopted. The orientation angles of the slits are set to be $\alpha=-\pi / 4$ and $\alpha=\pi / 4$, which leads to a dynamical PB phase $2 \sigma_{ \pm} \alpha$. A geometrical phase $k_{s p} s$ which is independent of incident polarization is introduced by spacing the slits a distance $s=\lambda_{s p} / 4$ apart. Thus, the phase of leftward and rightward SPP are $\varphi_{s p}^{l}=2 \sigma_{ \pm} \alpha+k_{s p} s$ and $\varphi_{s p}^{r}=2 \sigma_{ \pm} \alpha-k_{s p} s$, respectively. For LCP incident light, only the SPP propagating to the left satisfies the required phase modulation and SPP Airy beam is generated on the left side. When the incident light is switched to RCP, the SPP Airy beam is only observed on the right side. 
(a)
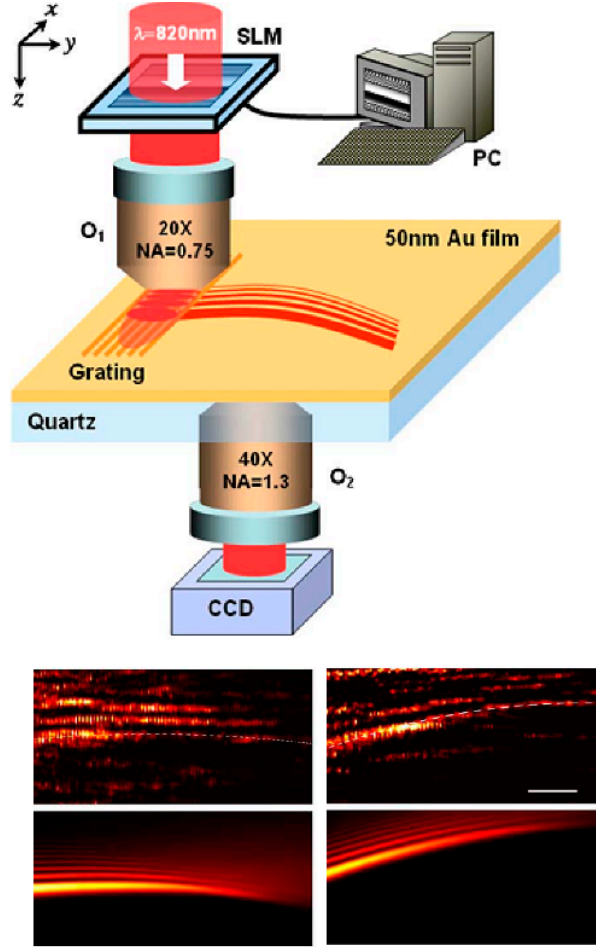

(b)
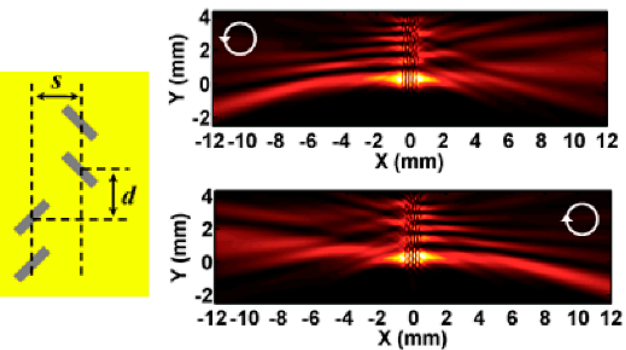

(c)
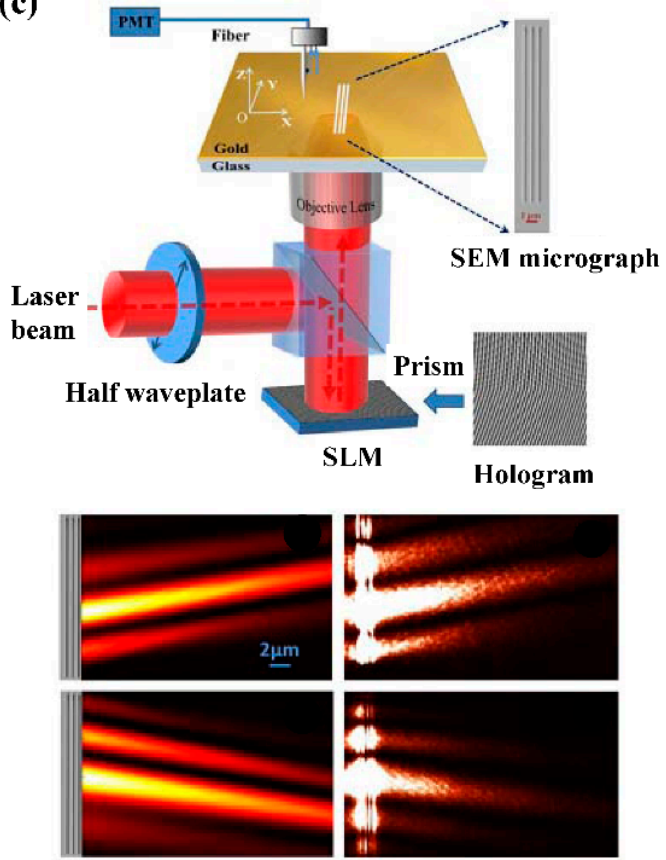

(d)
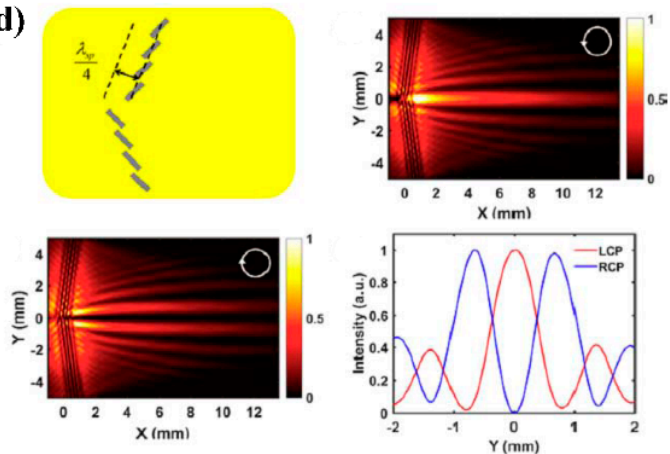

Figure 6. (a) Dynamic modulation of the trajectory of SPP Airy beam with SLM [42]; (b) Controlling the position of the SPP Airy beam by varying the incident polarization [74]; (c) Dynamic SPP Bessel beam through phase control [41]; (d) Polarization-based manipulation of SPP Bessel beams [75]. Reproduced with permissions from: [42], OSA, 2011, [74], OSA, 2017, [41], OSA, 2014, [75], OSA, 2018.

Compared with the parabolic profile of the SPP Airy beam, the trajectory of SPP Bessel beam is linear. Initial generations of SPP Bessel beam took advantage of intersecting grating structures which can excite two plane SPP waves with an inclined wavefront $[28,30]$. Such a SPP phase distribution can also be achieved by projecting a designed hologram onto a simple metallic grating [41]. Schematic diagram of the experimental setup is presented in Figure 6c. To excite SPP Bessel beam, a pure phase distribution $\varphi(x, y)=-k_{0}(|y| \sin \alpha+y \sin \beta)$ is addressed on the SLM. The width of the main lobe and the propagation direction of SPP Bessel beam can be manipulated by varying the parameters $\alpha$ and $\beta$, respectively. Breaking the intersecting grating into subwavelength slits, SPP Bessel beam with different profiles can be obtained by changing incident polarization [75]. As shown in Figure 6d, the orientation angles of upper and lower slits are $\alpha=\pi / 4$ and $\alpha=-\pi / 4$, repetitively. There also exists a $\lambda_{s p} / 4$ distance between the upper and lower slits. The phase difference of SPP generated by the upper and lower slits is $\Delta \varphi_{s p}=2 \sigma_{ \pm} \alpha-k_{s p} \lambda_{s p} / 4$. With the incident of LCP light, the SPP generated by the upper and lower slits are in phase $\left(\Delta \varphi_{s p}=0\right)$ and interfere constructively along the $x$-axis. For RCP incident light, the SPP generated by the upper and lower slits are out of phase $\left(\Delta \varphi_{s p}=\pi\right)$ and interfere destructively. Therefore, SPP beams with profiles of the zeroth-order Bessel function and the first-order Bessel function can be observed for LCP and RCP light, respectively. 


\section{Discussion and Perspectives}

Since the pioneering works of polarization-controlled SPP excitation by the F. Capasso group [48] and S. Zhang group [49], active SPP focusing by the E. Hasman group [57] and B. Gjonaj group [36], dynamic switching of SPP vortex by the B. Lee group [21] and Q. Zhan group [70], and dynamically controlled nondiffracting SPP beams by the X. Zhang Group [42], many researchers have devoted efforts to dynamic modulation of SPP and lots of fascinating works have been accomplished. We have briefly reviewed some of the achievements in the domain of dynamical SPP manipulation. According to the functionality, in this review, the dynamical SPP devices are classified in four categories: SPP excitation, SPP focusing, SPP vortex, and SPP nondiffracting beam. Other functionalities, such as dynamical SPP holography $[77,78]$ and controlled Cherenkov SPP wakes $[47,93]$, are demonstrated as well. Overall, modulating the wavelength, amplitude, phase, and polarization of the excitation light is the basic underlying principle of most dynamical SPP devices. The amplitude and phase can be controlled with SLMs and the wavelength-based modulation usually requires broadband light sources, which are complex and costly. On the contrary, the polarization states of light can be easily adjusted with half wave plates and quarter wave plates. With carefully designed subwavelength structures, almost all the functionalities of the SPP devices can be manipulated through polarization. Therefore, modulating the polarization of excitation light is a more convenient and effective way to actively control SPP field. Currently, most polarization-controlled SPP devices rely on changing the handedness of circularly polarized light. Further study may take advantage of linearly polarized light, elliptically polarized light, or light with space-variant state of polarization.

Coherence is also one of the fundamental degrees of freedom of light [94,95]. In the above discussion, we have mainly focused on the modulation of monochromatic SPP field. The spatial coherence of monochromatic SPP is conserved during propagation [96,97]. Theories for partially coherent polychromatic SPP field have been developed recently [98-103]. The SPP coherence characteristics can be actively controlled by tailoring the spectral and spatial coherence of incident light [98]. Particularly, Y. Chen et al. introduced and studied a class of structured polychromatic SPP field-partially coherent axiconic SPP field [100,101]. Theoretical analyses show that vectorial axiconic SPP field possesses high structural stability and broad statistical versatility. Despite the lack of interference for the uncorrelated axiconic SPP field, coherence lattices of varying forms can be obtained due to statistical similarity. These studies indicate that SPP coherence engineering is of fundamental importance in controlling the spatial, temporal, and polarization properties of the SPP field.

Besides, 2D materials such as graphene and $\mathrm{MoS}_{2}$ whose optical properties can be electrically tuned have been introduced as a new platform to dynamically modulate SPP [104-107]. The strong coupling between plasmon and material excitons [108-110] has been applied to plasmon enhanced spectroscopy. Combining the 2D materials, the modulation of excitation light and the design of subwavelength structure should enable a more flexible control over SPP fields in the future. Compared with static SPP devices, dynamical SPP devices are multifunctional and more efficient, which makes them a powerful tool in applications like on-chip communications $[10,11,83,111]$, polarization analyses $[70,112,113]$, particle manipulation $[69,114,115]$, and super resolution imaging $[38,116]$.

Author Contributions: This manuscript is written by X.L. and S.W. The discussions and revisions of the manuscript are carried out by X.L., C.Z., and S.W. All authors read and approved the final manuscript.

Funding: This work is supported by the National Natural Science Foundation of China (grant nos. 11704231, 11804199), China Postdoctoral Science Foundation (2017M622252).

Conflicts of Interest: The authors declare no conflict of interest.

\section{References}

1. Maier, S.A. Plasmonics: Fundamentals and Applications; Springer: New York, NY, USA, 2007.

2. Barnes, W.L.; Dereux, A.; Ebbesen, T.W. Surface plasmon subwavelength optics. Nature 2003, 424, 824-830.

[CrossRef] [PubMed] 
3. Zayats, A.V.; Smolyaninov, I.I.; Maradudin, A.A. Nano-optics of surface plasmon polaritons. Phys. Rep. 2005, 408, 131-314. [CrossRef]

4. Anker, J.N.; Hall, W.P.; Lyandres, O.; Shah, N.C.; Zhao, J.; Van Duyne, R.P. Biosensing with plasmonic nanosensors. Nat. Mater. 2008, 7, 442-453. [CrossRef] [PubMed]

5. Pillai, S.; Catchpole, K.R.; Trupke, T.; Green, M.A. Surface plasmon enhanced silicon solar cells. J. Appl. Phys. 2007, 101, 093105. [CrossRef]

6. Schuller, J.A.; Barnard, E.S.; Cai, W.; Jun, Y.C.; White, J.S.; Brongersma, M.L. Plasmonics for extreme light concentration and manipulation. Nat. Mater. 2010, 9, 193-204. [CrossRef] [PubMed]

7. Fang, N.; Lee, H.; Sun, C.; Zhang, X. Sub-diffraction-limited optical imaging with a silver superlens. Science 2005, 308, 534-537. [CrossRef] [PubMed]

8. Gramotnev, D.K.; Bozhevolnyi, S.I. Plasmonics beyond the diffraction limit. Nat. Photonics 2010, 4, 83-91. [CrossRef]

9. Sorger, V.J.; Oulton, R.F.; Ma, R.-M.; Zhang, X. Toward integrated plasmonic circuits. MRS Bull. 2012, 37, 728-738. [CrossRef]

10. Liu, Y.; Zhang, J.; Liu, H.; Wang, S.; Peng, L.M. Electrically driven monolithic subwavelength plasmonic interconnect circuits. Sci. Adv. 2017, 3, e1701456. [CrossRef]

11. Fukuda, M.; Tonooka, Y.; Inoue, T.; Ota, M. Feasibility of plasmonic circuits for on-chip interconnects. Solid State Electron. 2019, 156, 33-40. [CrossRef]

12. Zentgraf, T.; Liu, Y.; Mikkelsen, M.H.; Valentine, J.; Zhang, X. Plasmonic Luneburg and Eaton lenses. Nat. Nanotechnol. 2011, 6, 151-155. [CrossRef]

13. Yin, L.; Vlasko-Vlasov, V.K.; Pearson, J.; Hiller, J.M.; Hua, J.; Welp, U.; Brown, D.E.; Kimball, C.W. Subwavelength focusing and guiding of surface plasmons. Nano Lett. 2005, 5, 1399-1402. [CrossRef]

14. Lerman, G.M.; Yanai, A.; Levy, U. Demonstration of Nanofocusing by the use of Plasmonic Lens Illuminated with Radially Polarized Light. Nano Lett. 2009, 9, 2139-2143. [CrossRef]

15. Fang, Z.; Peng, Q.; Song, W.; Hao, F.; Wang, J.; Nordlander, P.; Zhu, X. Plasmonic focusing in symmetry broken nanocorrals. Nano Lett. 2011, 11, 893-897. [CrossRef]

16. Li, G.; Sun, Y.; Wang, S. Spin-Independent Plasmonic Lens. Nanoscale Res. Lett. 2019, 14, 156. [CrossRef]

17. Li, L.; Li, T.; Wang, S.; Zhu, S.; Zhang, X. Broadband focusing and demultiplexing of in-plane propagating surface plasmons. Nano Lett. 2011, 11, 4357-4361. [CrossRef]

18. Ditlbacher, H.; Krenn, J.R.; Schider, G.; Leitner, A.; Aussenegg, F.R. Two-dimensional optics with surface plasmon polaritons. Appl. Phys. Lett. 2002, 81, 1762-1764. [CrossRef]

19. Randhawa, S.; Gonzalez, M.U.; Renger, J.; Enoch, S.; Quidant, R. Design and properties of dielectric surface plasmon Bragg mirrors. Opt. Express 2010, 18, 14496-14510. [CrossRef]

20. Gonzalez, M.U.; Weeber, J.C.; Baudrion, A.L.; Dereux, A.; Stepanov, A.L.; Krenn, J.R.; Devaux, E.; Ebbesen, T.W. Design, near-field characterization, and modeling of 45 circle surface-plasmon Bragg mirrors. Phys. Rev. B 2006, 73, 155416. [CrossRef]

21. Kim, H.; Park, J.; Cho, S.W.; Lee, S.Y.; Kang, M.; Lee, B. Synthesis and dynamic switching of surface plasmon vortices with plasmonic vortex lens. Nano Lett. 2010, 10, 529-536. [CrossRef]

22. Li, X.; Gao, Y.; Jiang, S.; Ma, L.; Liu, C.; Cheng, C. Experimental solution for scattered imaging of the interference of plasmonic and photonic mode waves launched by metal nano-slits. Opt. Express 2015, 23, 3507-3522. [CrossRef]

23. Wang, H.; Liu, L.; Liu, C.; Li, X.; Wang, S.; Xu, Q.; Teng, S. Plasmonic vortex generator without polarization dependence. New J. Phys. 2018, 20, 033024. [CrossRef]

24. Zhang, Y.; Zhang, R.; Li, X.; Ma, L.; Liu, C.; He, C.; Cheng, C. Radially polarized plasmonic vector vortex generated by a metasurface spiral in gold film. Opt. Express 2017, 25, 32150-32160. [CrossRef]

25. Minovich, A.; Klein, A.E.; Janunts, N.; Pertsch, T.; Neshev, D.N.; Kivshar, Y.S. Generation and near-field imaging of Airy surface plasmons. Phys. Rev. Lett. 2011, 107, 116802. [CrossRef]

26. Li, L.; Li, T.; Wang, S.M.; Zhang, C.; Zhu, S.N. Plasmonic Airy beam generated by in-plane diffraction. Phys. Rev. Lett. 2011, 107, 126804. [CrossRef]

27. Kou, S.S.; Yuan, G.; Wang, Q.; Du, L.; Balaur, E.; Zhang, D.; Tang, D.; Abbey, B.; Yuan, X.-C.; Lin, J. On-chip photonic Fourier transform with surface plasmon polaritons. Light Sci. Appl. 2016, 5, e16034. [CrossRef]

28. Lin, J.; Dellinger, J.; Genevet, P.; Cluzel, B.; de Fornel, F.; Capasso, F. Cosine-Gauss plasmon beam: A localized long-range nondiffracting surface wave. Phys. Rev. Lett. 2012, 109, 093904. [CrossRef] 
29. Li, L.; Li, T.; Wang, S.M.; Zhu, S.N. Collimated plasmon beam: Nondiffracting versus linearly focused. Phys. Rev. Lett. 2013, 110, 046807. [CrossRef]

30. Garcia-Ortiz, C.E.; Coello, V.; Han, Z.; Bozhevolnyi, S.I. Generation of diffraction-free plasmonic beams with one-dimensional Bessel profiles. Opt. Lett. 2013, 38, 905-907. [CrossRef]

31. Fu, Y.; Hu, X.; Lu, C.; Yue, S.; Yang, H.; Gong, Q. All-optical logic gates based on nanoscale plasmonic slot waveguides. Nano Lett. 2012, 12, 5784-5790. [CrossRef]

32. Lu, C.C.; Hu, X.Y.; Yang, H.; Gong, Q.H. Integrated all-optical logic discriminators based on plasmonic bandgap engineering. Sci. Rep. 2013, 3, 2778. [CrossRef]

33. Baron, A.; Devaux, E.; Rodier, J.C.; Hugonin, J.P.; Rousseau, E.; Genet, C.; Ebbesen, T.W.; Lalanne, P. Compact Antenna for Efficient and Unidirectional Launching and Decoupling of Surface Plasmons. Nano Lett. 2011, 11, 4207-4212. [CrossRef]

34. Lopez-Tejeira, F.; Rodrigo, S.G.; Martin-Moreno, L.; Garcia-Vidal, F.J.; Devaux, E.; Ebbesen, T.W.; Krenn, J.R.; Radko, I.P.; Bozhevolnyi, S.I.; Gonzalez, M.U.; et al. Efficient unidirectional nanoslit couplers for surface plasmons. Nat. Phys. 2007, 3, 324-328. [CrossRef]

35. Chen, J.; Li, Z.; Yue, S.; Gong, Q. Efficient unidirectional generation of surface plasmon polaritons with asymmetric single-nanoslit. Appl. Phys. Lett. 2010, 97, 041113. [CrossRef]

36. Gjonaj, B.; Aulbach, J.; Johnson, P.M.; Mosk, A.P.; Kuipers, L.; Lagendijk, A. Active spatial control of plasmonic fields. Nat. Photonics 2011, 5, 360-363. [CrossRef]

37. Zhao, C.; Zhang, J. Flexible wavefront manipulation of surface plasmon polaritons without mechanical motion components. Appl. Phys. Lett. 2011, 98, 211108. [CrossRef]

38. Gjonaj, B.; Aulbach, J.; Johnson, P.M.; Mosk, A.P.; Kuipers, L.; Lagendijk, A. Focusing and scanning microscopy with propagating surface plasmons. Phys. Rev. Lett. 2013, 110, 266804. [CrossRef]

39. Yuan, G.H.; Wang, Q.; Tan, P.S.; Lin, J.; Yuan, X.C. A dynamic plasmonic manipulation technique assisted by phase modulation of an incident optical vortex beam. Nanotechnology 2012, 23, 385204. [CrossRef]

40. Yuan, G.H.; Yuan, X.C.; Bu, J.; Tan, P.S.; Wang, Q. Manipulation of surface plasmon polaritons by phase modulation of incident light. Opt. Express 2011, 19, 224-229. [CrossRef]

41. Xiao, K.; Wei, S.; Min, C.; Yuan, G.; Zhu, S.W.; Lei, T.; Yuan, X.C. Dynamic cosine-Gauss plasmonic beam through phase control. Opt. Express 2014, 22, 13541-13546. [CrossRef]

42. Zhang, P.; Wang, S.; Liu, Y.; Yin, X.; Lu, C.; Chen, Z.; Zhang, X. Plasmonic Airy beams with dynamically controlled trajectories. Opt. Lett. 2011, 36, 3191-3193. [CrossRef]

43. Wei, S.; Si, G.; Malek, M.; Earl, S.K.; Du, L.; Kou, S.S.; Yuan, X.; Lin, J. Toward broadband, dynamic structuring of a complex plasmonic field. Sci. Adv. 2018, 4, eaao0533. [CrossRef]

44. Cho, S.W.; Park, J.; Lee, S.Y.; Kim, H.; Lee, B. Coupling of spin and angular momentum of light in plasmonic vortex. Opt. Express 2012, 20, 10083-10094. [CrossRef]

45. Gjonaj, B.; Aulbach, J.; Johnson, P.M.; Mosk, A.P.; Kuipers, L.; Lagendijk, A. Optical control of plasmonic Bloch modes on periodic nanostructures. Nano Lett. 2012, 12, 546-550. [CrossRef]

46. Lee, S.; Kim, K.; Kim, S.; Park, H.; Kim, K.; Lee, B. Plasmonic meta-slit: Shaping and controlling near-field focus. Optica 2015, 2, 6-13. [CrossRef]

47. Genevet, P.; Wintz, D.; Ambrosio, A.; She, A.; Blanchard, R.; Capasso, F. Controlled steering of Cherenkov surface plasmon wakes with a one-dimensional metamaterial. Nat. Nanotechnol. 2015, 10, 804-809. [CrossRef]

48. Lin, J.; Mueller, J.P.; Wang, Q.; Yuan, G.; Antoniou, N.; Yuan, X.C.; Capasso, F. Polarization-controlled tunable directional coupling of surface plasmon polaritons. Science 2013, 340, 331-334. [CrossRef]

49. Huang, L.; Chen, X.; Bai, B.; Tan, Q.; Jin, G.; Zentgraf, T.; Zhang, S. Helicity dependent directional surface plasmon polariton excitation using a metasurface with interfacial phase discontinuity. Light Sci. Appl. 2013, 2, e70. [CrossRef]

50. Lefier, Y.; Salut, R.; Suarez, M.A.; Grosjean, T. Directing Nanoscale Optical Flows by Coupling Photon Spin to Plasmon Extrinsic Angular Momentum. Nano Lett. 2018, 18, 38-42. [CrossRef]

51. Jin, J.; Li, X.; Guo, Y.; Pu, M.; Gao, P.; Ma, X.; Luo, X. Polarization-controlled unidirectional excitation of surface plasmon polaritons utilizing catenary apertures. Nanoscale 2019, 11, 3952-3957. [CrossRef]

52. Xu, Q.; Zhang, X.; Yang, Q.; Tian, C.; Xu, Y.; Zhang, J.; Zhao, H.; Li, Y.; Ouyang, C.; Tian, Z.; et al. Polarization-controlled asymmetric excitation of surface plasmons. Optica 2017, 4, 1044-1051. [CrossRef]

53. Zhang, X.; Xu, Y.; Yue, W.; Tian, Z.; Gu, J.; Li, Y.; Singh, R.; Zhang, S.; Han, J.; Zhang, W. Anomalous Surface Wave Launching by Handedness Phase Control. Adv. Mater. 2015, 27, 7123-7129. [CrossRef] 
54. Pan, D.; Wei, H.; Gao, L.; Xu, H. Strong Spin-Orbit Interaction of Light in Plasmonic Nanostructures and Nanocircuits. Phys. Rev. Lett. 2016, 117, 166803. [CrossRef]

55. Mueller, J.P.; Leosson, K.; Capasso, F. Polarization-selective coupling to long-range surface plasmon polariton waveguides. Nano Lett. 2014, 14, 5524-5527. [CrossRef]

56. Pors, A.; Nielsen, M.G.; Bernardin, T.; Weeber, J.-C.; Bozhevolnyi, S.I. Efficient unidirectional polarization-controlled excitation of surface plasmon polaritons. Light Sci. Appl. 2014, 3, e197. [CrossRef]

57. Bliokh, K.Y.; Gorodetski, Y.; Kleiner, V.; Hasman, E. Coriolis effect in optics: Unified geometric phase and spin-Hall effect. Phys. Rev. Lett. 2008, 101, 030404. [CrossRef]

58. Gorodetski, Y.; Niv, A.; Kleiner, V.; Hasman, E. Observation of the spin-based plasmonic effect in nanoscale structures. Phys. Rev. Lett. 2008, 101, 043903. [CrossRef]

59. Shitrit, N.; Nechayev, S.; Kleiner, V.; Hasman, E. Spin-dependent plasmonics based on interfering topological defects. Nano Lett. 2012, 12, 1620-1623. [CrossRef]

60. Wang, S.; Wang, X.K.; Zhao, F.; Qu, S.L.; Zhang, Y. Observation and explanation of polarization-controlled focusing of terahertz surface plasmon polaritons. Phys. Rev. A 2015, 91, 053812. [CrossRef]

61. Bao, Y.; Zu, S.; Liu, W.; Zhou, L.; Zhu, X.; Fang, Z. Revealing the spin optics in conic-shaped metasurfaces. Phys. Rev. B 2017, 95, 081406. [CrossRef]

62. Sun, Y.; Zhao, C.; Li, G.; Li, X.; Wang, S. Enlarging spin-dependent transverse displacement of surface plasmon polaritons focus. Opt. Express 2019, 27, 11112-11121. [CrossRef]

63. Lee, S.Y.; Kim, K.; Lee, G.Y.; Lee, B. Polarization-multiplexed plasmonic phase generation with distributed nanoslits. Opt. Express 2015, 23, 15598-15607. [CrossRef]

64. Wintz, D.; Genevet, P.; Ambrosio, A.; Woolf, A.; Capasso, F. Holographic metalens for switchable focusing of surface plasmons. Nano Lett. 2015, 15, 3585-3589. [CrossRef]

65. Man, Z.; Du, L.; Min, C.; Zhang, Y.; Zhang, C.; Zhu, S.; Paul Urbach, H.; Yuan, X.C. Dynamic plasmonic beam shaping by vector beams with arbitrary locally linear polarization states. Appl. Phys. Lett. 2014, 105, 011110. [CrossRef]

66. Li, X.; Zhang, R.; Zhang, Y.; Ma, L.; He, C.; Ren, X.; Liu, C.; Cheng, C. Slit width oriented polarized wavefields transition involving plasmonic and photonic modes. New J. Phys. 2018, 20, 063037. [CrossRef]

67. Lee, S.-Y.; Kim, S.-J.; Kwon, H.; Lee, B. Spin-Direction Control of High-Order Plasmonic Vortex with Double-Ring Distributed Nanoslits. IEEE Photonics Technol. Lett. 2015, 27, 705-708. [CrossRef]

68. Liu, A.; Rui, G.; Ren, X.; Zhan, Q.; Guo, G.; Guo, G. Encoding photonic angular momentum information onto surface plasmon polaritons with plasmonic lens. Opt. Express 2012, 20, 24151-24159. [CrossRef]

69. Tsai, W.Y.; Huang, J.S.; Huang, C.B. Selective trapping or rotation of isotropic dielectric microparticles by optical near field in a plasmonic archimedes spiral. Nano Lett. 2014, 14, 547-552. [CrossRef]

70. Chen, W.; Abeysinghe, D.C.; Nelson, R.L.; Zhan, Q. Experimental confirmation of miniature spiral plasmonic lens as a circular polarization analyzer. Nano Lett. 2010, 10, 2075-2079. [CrossRef]

71. Zhang, Y.Q.; Zeng, X.Y.; Zhang, R.R.; Zhan, Z.J.; Li, X.; Ma, L.; Liu, C.X.; He, C.W.; Cheng, C.F. Generation of a plasmonic radially polarized vector beam with linearly polarized illumination. Opt. Lett. 2018, 43, 4208-4211. [CrossRef]

72. Zhang, Q.; Wang, H.; Liu, L.; Teng, S. Generation of vector beams using spatial variation nanoslits with linearly polarized light illumination. Opt. Express 2018, 26, 24145-24153. [CrossRef]

73. Zang, X.; Zhu, Y.; Mao, C.; Xu, W.; Ding, H.; Xie, J.; Cheng, Q.; Chen, L.; Peng, Y.; Hu, Q.; et al. Manipulating Terahertz Plasmonic Vortex Based on Geometric and Dynamic Phase. Adv. Opt. Mater. 2018, 7, 1801328. [CrossRef]

74. Wang, S.; Wang, X.; Zhang, Y. Simultaneous Airy beam generation for both surface plasmon polaritons and transmitted wave based on metasurface. Opt. Express 2017, 25, 23589-23596. [CrossRef]

75. Wang, S.; Wang, S.; Zhang, Y. Polarization-based dynamic manipulation of Bessel-like surface plasmon polaritons beam. Opt. Express 2018, 26, 5461-5468. [CrossRef]

76. Avayu, O.; Epstein, I.; Eizner, E.; Ellenbogen, T. Polarization controlled coupling and shaping of surface plasmon polaritons by nanoantenna arrays. Opt. Lett. 2015, 40, 1520-1523. [CrossRef]

77. Xiao, S.; Zhong, F.; Liu, H.; Zhu, S.; Li, J. Flexible coherent control of plasmonic spin-Hall effect. Nat. Commun. 2015, 6, 8360. [CrossRef]

78. Xu, Q.; Zhang, X.; Xu, Y.; Ouyang, C.; Tian, Z.; Gu, J.; Li, J.; Zhang, S.; Han, J.; Zhang, W. Polarization-controlled surface plasmon holography. Laser Photonics Rev. 2017, 11, 1600212. [CrossRef] 
79. Liu, J.S.; Pala, R.A.; Afshinmanesh, F.; Cai, W.; Brongersma, M.L. A submicron plasmonic dichroic splitter. Nat. Commun. 2011, 2, 525. [CrossRef]

80. Yao, W.; Liu, S.; Liao, H.; Li, Z.; Sun, C.; Chen, J.; Gong, Q. Efficient directional excitation of surface plasmons by a single-element nanoantenna. Nano Lett. 2015, 15, 3115-3121. [CrossRef]

81. Tanemura, T.; Balram, K.C.; Ly-Gagnon, D.S.; Wahl, P.; White, J.S.; Brongersma, M.L.; Miller, D.A. Multiple-wavelength focusing of surface plasmons with a nonperiodic nanoslit coupler. Nano Lett. 2011, 11, 2693-2698. [CrossRef]

82. Chen, J.; Chen, X.; Li, T.; Zhu, S. On-Chip Detection of Orbital Angular Momentum Beam by Plasmonic Nanogratings. Laser Photonics Rev. 2018, 12, 1700331. [CrossRef]

83. Mei, S.; Huang, K.; Liu, H.; Qin, F.; Mehmood, M.Q.; Xu, Z.; Hong, M.; Zhang, D.; Teng, J.; Danner, A.; et al. On-chip discrimination of orbital angular momentum of light with plasmonic nanoslits. Nanoscale 2016, 8 , 2227-2233. [CrossRef]

84. Liu, Z.; Steele, J.M.; Lee, H.; Zhang, X. Tuning the focus of a plasmonic lens by the incident angle. Appl. Phys. Lett. 2006, 88, 171108. [CrossRef]

85. Qiu, P.; Zhang, D.; Jing, M.; Lu, T.; Yu, B.; Zhan, Q.; Zhuang, S. Dynamic tailoring of surface plasmon polaritons through incident angle modulation. Opt. Express 2018, 26, 9772-9783. [CrossRef]

86. Drezet, A.; Koller, D.; Hohenau, A.; Leitner, A.; Aussenegg, F.R.; Krenn, J.R. Plasmonic crystal demultiplexer and multiports. Nano Lett. 2007, 7, 1697-1700. [CrossRef]

87. Muskens, O.L.; Del Fatti, N.; Vallee, F. Femtosecond response of a single metal nanoparticle. Nano Lett. 2006, 6, 552-556. [CrossRef]

88. Pelton, M.; Liu, M.; Park, S.; Scherer, N.F.; Guyot-Sionnest, P. Ultrafast resonant optical scattering from single gold nanorods: Large nonlinearities and plasmon saturation. Phys. Rev. B 2006, 73, 155419. [CrossRef]

89. Allen, L.; Beijersbergen, M.W.; Spreeuw, R.J.; Woerdman, J.P. Orbital angular momentum of light and the transformation of Laguerre-Gaussian laser modes. Phys. Rev. A 1992, 45, 8185-8189. [CrossRef]

90. Beijersbergen, M.W.; Coerwinkel, R.P.C.; Kristensen, M.; Woerdman, J.P. Helical wavefront laser beams produced with a spiral phaseplate. Opt. Commun. 1994, 112, 321-327. [CrossRef]

91. Chen, J.; Li, Z.; Zhang, X.; Xiao, J.; Gong, Q. Submicron bidirectional all-optical plasmonic switches. Sci. Rep. 2013, 3, 1451. [CrossRef]

92. Zhao, C.; Liu, Y.; Zhao, Y.; Fang, N.; Huang, T.J. A reconfigurable plasmofluidic lens. Nat. Commun. 2013, 4, 2305. [CrossRef]

93. Sain, B.; Kaner, R.; Prior, Y. Phase-controlled propagation of surface plasmons. Light Sci. Appl. 2017, 6, e17072. [CrossRef]

94. Morrill, D.; Li, D.; Pacifici, D. Measuring subwavelength spatial coherence with plasmonic interferometry. Nat. Photonics 2016, 10, 681-687. [CrossRef]

95. Divitt, S.; Frimmer, M.; Visser, T.D.; Novotny, L. Modulation of optical spatial coherence by surface plasmon polaritons. Opt. Lett. 2016, 41, 3094-3097. [CrossRef]

96. Guebrou, S.A.; Laverdant, J.; Symonds, C.; Vignoli, S.; Bellessa, J. Spatial coherence properties of surface plasmon investigated by Young's slit experiment. Opt. Lett. 2012, 37, 2139-2141. [CrossRef]

97. Laverdant, J.; AberraGuebrou, S.; Bessueille, F.; Symonds, C.; Bellessa, J. Leakage interferences applied to surface plasmon analysis. J. Opt. Soc. Am. A 2014, 31, 1067-1073. [CrossRef]

98. Norrman, A.; Ponomarenko, S.A.; Friberg, A.T. Partially coherent surface plasmon polaritons. Europhys. Lett. 2016, 116, 64001. [CrossRef]

99. Chen, Y.; Norrman, A.; Ponomarenko, S.A.; Friberg, A.T. Plasmon coherence determination by nanoscattering. Opt. Lett. 2017, 42, 3279-3282. [CrossRef]

100. Chen, Y.; Norrman, A.; Ponomarenko, S.A.; Friberg, A.T. Partially coherent axiconic surface plasmon polariton fields. Phys. Rev. A 2018, 97, 041801. [CrossRef]

101. Chen, Y.; Norrman, A.; Ponomarenko, S.A.; Friberg, A.T. Coherence lattices in surface plasmon polariton fields. Opt. Lett. 2018, 43, 3429-3432. [CrossRef]

102. Mao, H.; Chen, Y.; Ponomarenko, S.A.; Friberg, A.T. Coherent pseudo-mode representation of partially coherent surface plasmon polaritons. Opt. Lett. 2018, 43, 1395-1398. [CrossRef]

103. Daniel, S.; Saastamoinen, K.; Ponomarenko, S.A.; Friberg, A.T. Scattering of partially coherent surface plasmon polariton fields by metallic nanostripe. J. Eur. Opt. Soc. 2019, 15, 4. [CrossRef] 
104. He, X.; Liu, F.; Lin, F.; Xiao, G.; Shi, W. Tunable MoS2 modified hybrid surface plasmon waveguides. Nanotechnology 2019, 30, 125201. [CrossRef]

105. Li, R.; Imran, M.; Lin, X.; Wang, H.; Xu, Z.; Chen, H. Hybrid Airy plasmons with dynamically steerable trajectories. Nanoscale 2017, 9, 1449-1456. [CrossRef]

106. Gao, W.; Shi, G.; Jin, Z.; Shu, J.; Zhang, Q.; Vajtai, R.; Ajayan, P.M.; Kono, J.; Xu, Q. Excitation and active control of propagating surface plasmon polaritons in graphene. Nano Lett. 2013, 13, 3698-3702. [CrossRef]

107. Yang, Y.; Dai, H.T.; Zhu, B.F.; Sun, X.W. Dynamic Control of the Airy Plasmons in a Graphene Platform. IEEE Photonics J. 2014, 6, 1-7. [CrossRef]

108. Dintinger, J.; Klein, S.; Bustos, F.; Barnes, W.L.; Ebbesen, T.W. Strong coupling between surface plasmon-polaritons and organic molecules in subwavelength hole arrays. Phys. Rev. B 2005, 71, 035424. [CrossRef]

109. Christ, A.; Tikhodeev, S.G.; Gippius, N.A.; Kuhl, J.; Giessen, H. Waveguide-plasmon polaritons: Strong coupling of photonic and electronic resonances in a metallic photonic crystal slab. Phys. Rev. Lett. 2003, 91, 183901. [CrossRef]

110. Itoh, T.; Yamamoto, Y.S. Reproduction of surface-enhanced resonant Raman scattering and fluorescence spectra of a strong coupling system composed of a single silver nanoparticle dimer and a few dye molecules. J. Chem. Phys. 2018, 149, 244701. [CrossRef]

111. Genevet, P.; Lin, J.; Kats, M.A.; Capasso, F. Holographic detection of the orbital angular momentum of light with plasmonic photodiodes. Nat. Commun. 2012, 3, 1278. [CrossRef]

112. Lerman, G.M.; Levy, U. Pin cushion plasmonic device for polarization beam splitting, focusing, and beam position estimation. Nano Lett. 2013, 13, 1100-1105. [CrossRef]

113. Wang, S.; Wang, X.; Kan, Q.; Qu, S.; Zhang, Y. Circular polarization analyzer with polarization tunable focusing of surface plasmon polaritons. Appl. Phys. Lett. 2015, 107, 243504. [CrossRef]

114. Spektor, G.; Kilbane, D.; Mahro, A.K.; Frank, B.; Ristok, S.; Gal, L.; Kahl, P.; Podbiel, D.; Mathias, S.; Giessen, H.; et al. Revealing the subfemtosecond dynamics of orbital angular momentum in nanoplasmonic vortices. Science 2017, 355, 1187-1191. [CrossRef]

115. Shen, Z.; Hu, Z.J.; Yuan, G.H.; Min, C.J.; Fang, H.; Yuan, X.C. Visualizing orbital angular momentum of plasmonic vortices. Opt. Lett. 2012, 37, 4627-4629. [CrossRef]

116. Ertsgaard, C.T.; McKoskey, R.M.; Rich, I.S.; Lindquist, N.C. Dynamic placement of plasmonic hotspots for super-resolution surface-enhanced Raman scattering. ACS Nano 2014, 8, 10941-10946. [CrossRef]

(C) 2019 by the authors. Licensee MDPI, Basel, Switzerland. This article is an open access article distributed under the terms and conditions of the Creative Commons Attribution (CC BY) license (http://creativecommons.org/licenses/by/4.0/). 